\title{
Analysis of Breast Cancer Cell Subpopulations for the Expression and Distribution of HER2 and their Response to Anti-Cancer Drugs Using Electron Microscopy
}

\author{
$\underline{\text { Patricia Blach }^{1}}, \underline{\text { Florian Weinberg }^{1}}$, Diana Peckys ${ }^{2}$, Indra N. Dahmke ${ }^{1}$ and Niels de Jonge ${ }^{1,3}$ \\ ${ }^{1}$ INM - Leibniz Institute for New Materials, D-66123 Saarbrücken, Germany \\ ${ }^{2}$ Department of Biophysics, Saarland University, D-66421 Homburg, Germany \\ ${ }^{3}$ Department of Physics, Saarland University, D-66123 Saarbrücken, Germany
}

Breast cancer is the leading cause of mortality in women worldwide and is divided in different subtypes according to the presence of growth factor receptors on the surface of cancer cells [1]. Around $20 \%$ of all breast cancer cases overexpress the growth factor receptor HER2 belonging to the EGFreceptor family [2]. Although HER2 positive breast cancer can be treated with drugs like neutralizing antibodies (Trastuzumab or Pertuzumab) or small compound inhibitors (Lapatinib), development of therapy resistances create huge problems in clinics [3].

We aim to understand the principles of drug action, like trastuzumab and resistance development on the single cell level. We are especially interested in understanding therapy resistance development for cancer cell subpopulations as individual cancer cells were shown to correspond differently towards therapy [4].

We analyzed HER2 on the surface of cancer cells in native liquid state conformation by labeling them 1:1 with affibodies followed by linking quantum dot nanoparticles [5] (Figure 1a). The physical properties of quantum dots allow fluorescent image analysis by bright field light microscopy, and also electron microscopy analysis with nanometer resolution.

For electron microscopic analyses, we employed liquid-phase scanning transmission electron microscopy (STEM) permitting visualization of labeled receptors either as single molecules or as receptor-pairs, so-called dimers, expressed on the cell surface. To this end, HER2 overexpressing breast cancer cells were seeded on a Si microchip with an electron transparent SiN window. After labeling of HER2 and fluorescence imaging, the cells were coated with a thin layer of graphene for maintaining a thin liquid layer when placed in the vacuum chamber of the electron microscope (Figure 1b). Exemplary STEM images are shown in Figure 2a/b with quantum dots visible as bright spots on the cell surface of a cancer cell. We used automatic detection scripts for ImageJ (Figure 2c) to determine the spatial distribution of HER2 molecules by pair correlation function analysis (Figure $2 \mathrm{~d}$ ), whereby higher values than 1 indicate an increased probability of a certain distance between two HER2 molecules to occur. We observed a sharp peak at $20 \mathrm{~nm}$ indicating the formation of HER2 homo-dimers.

Our established protocols further allow combination of the receptor labeling with classical immunofluorescent labeling approaches, which will be applied to identify different subpopulations of cancer cells, for instance cancer stem cells known to be involved in drug resistance $[2,4,6,8]$.

\section{References:}

[1] G Turashvili and E Brogi, Front Med 4 (2017), p. 227.

[2] B Martin-Castillo et al, Oncotarget 6 (2015), p. 32317.

[3] T Vu and FX Claret, Front Oncol 2 (2012), p. 62.

[4] DB Peckys et al, Mol Biol Cell 28 (2017), p. 3193.

[5] DB Peckys, U Korf and N de Jonge, Sci Adv 1 (2015), p. e1500165.

[6] B Nami and Z Wang, Cancers 9 (2017), p. 40. 
[7] IN Dahmke et al, ACS Nano 11 (2017), p. 11108.

[8] We thank Eduard Arzt for his support through INM. This work was funded by the Else KrönerFresenius Stiftung.

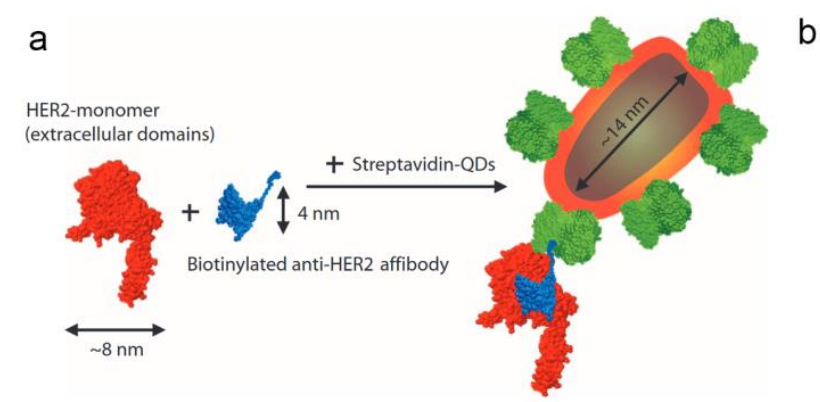

b
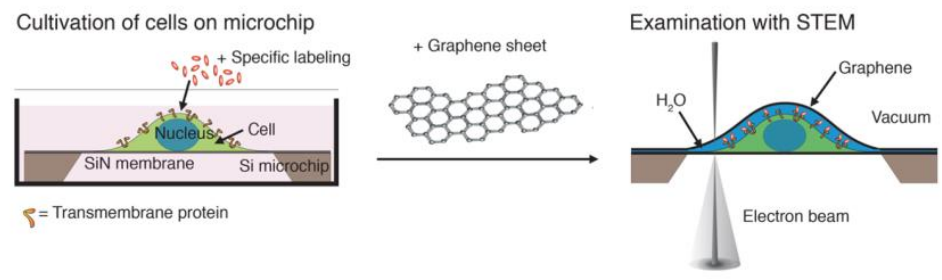

Figure 1: Principle of STEM of labeled membrane proteins in whole cells. a) Labeling of HER2 with a biotinylated HER2 affibody followed by the linkage to a streptavidin coated quantum dots. Figure from [5]. b) HER2 labeling is carried out in liquid followed by covering of the cells with a thin layer of graphene to maintain a saturated water atmosphere during STEM imaging. With permission from [7]. Copyright 2017 American Chemical Society.
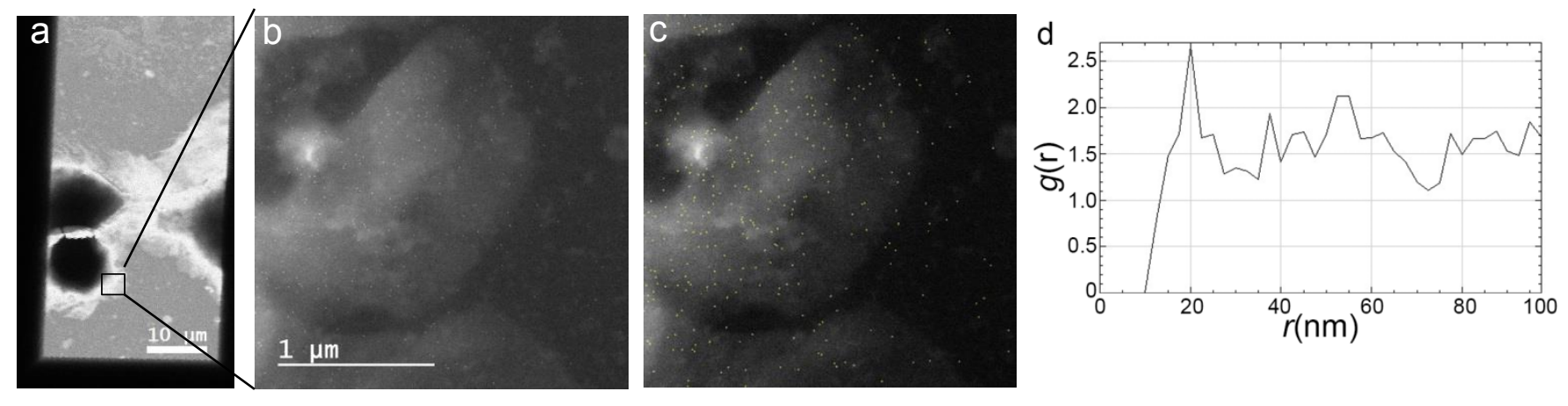

Figure 2: STEM of HER2 proteins in breast cancer cells. a) Exemplary STEM-Image at 800x and b) at $80000 \mathrm{x}$ magnification showing the abundance of quantum dot labeled HER2 molecules. c) Result of automatic detection of quantum dots (yellow spots). d) Pair correlation function analysis of label positions in c) indicating HER2 dimer formation at a distance of $20 \mathrm{~nm}$. 